\title{
Cross-finger Flap to the Thumb: Quest for an Alternate Donor
}

\author{
Madhavi Chitta $^{1} \quad$ Lekshmi Malathi $^{1, \odot}$ Aneesh Joseph ${ }^{1}$ \\ ${ }^{1}$ Department of Plastic Surgery, Government Medical College, \\ Gandhinagar, Kottayam, Kerala, India

\begin{abstract}
Address for correspondence Lekshmi Malathi, MS, M.Ch. (Plastic Surg.), DNB (Plastic Surg.), Department of Plastic Surgery, Government Medical College, Gandhinagar, Kottayam, Kerala, 686008, India (e-mail: jayakumar.lekshmi@gmail.com).
\end{abstract}

\begin{abstract}
Keywords

- Cross-finger flap

- donor finger

- ring finger

- thumb injury

- thumb reconstruction

Background Distal thumb injuries are managed by multiple local and regional flaps. The cross-finger flap (CFF) is one versatile flap used to cover such defects. The donor finger to the thumb is classically described to be the index finger (IF). However, with the index finger being the second most important finger, it causes further debilitation of the already injured hand. Our aim was to find an alternate donor finger to spare the index finger.

Methods and Materials A prospective observational study was conducted on 10 patients with distal thumb injuries who were admitted between November 2018 and July 2019. Ring finger (RF) was used as donor for CFF in all the cases. The subjects were assessed for first web space angle, Kapandji score, total range of movement (TRM) of long fingers, and global hand function using Michigan hand outcome questionnaire (MHQ) after flap division.

Results There was no first web contracture and TRM of IF remained unaffected. However, TRM of RF was significantly reduced. But this being a relatively unimportant finger, there was no significant reduction in global hand function, as evaluated by the MHQ score.

Conclusion The ring finger is a good alternative for index finger as donor for CFF to the thumb, because it provides adequate tissue along with comfortable positioning and easy maintenance of local hygiene. Moreover, it preserves global hand function by sparing the IF.
\end{abstract}

\section{Introduction}

"The hand without a thumb is at worst nothing but an animated fish slice, and at best a pair of forceps whose points don't meet properly"

-John Napier ${ }^{1}$

The thumb is a significant part of the hand contributing to approximately $40 \%$ of the function of the hand.

Cross-finger flaps (CFF) were first described by Gurdin and Pangman in 1950 and $1951 .^{2}$ These flaps were originally meant for volar defects of middle and terminal phalanges. Their use has gradually progressed to coverage of dorsal defects and amputation stumps. The CFF is advantageous in many ways, in that it is easy to perform, provides a reliable flap in view of the robust blood supply to the hand, and provides tactile gnostic ability and a good contour to the defect. ${ }^{3}$ The flap is commonly harvested from the adjacent finger due to its ease of positioning. The donor finger for CFF to thumb is classically the index finger (IF), the next most important finger of the hand. ${ }^{1,4}$ It is involved in the eight basic positions of the hand that make up most maneuvers. ${ }^{5}$
License terms

()(1) $\Theta \circledast$ 
The morbidity of donor finger includes unsightly donor scar, flexion deficit, stiffness, and impaired pinch grip strength in general. ${ }^{6,7}$ Our personal experience of three cases of CFF to thumb from IF showed, in addition, some amount of first web contracture and poor maintenance of hygiene with flap in situ.

The purpose of this study is to propose an alternate finger other than the IF as donor for CFF to the thumb, so that in the injuries to the thumb, the IF is spared of the above-mentioned impairments, thus preserving available global hand function.

\section{Materials and Methods}

A prospective observational study was conducted on 10 patients with soft tissue defects of thumb who attended the emergency department from November 2018 to July 2019. Defects proximal to the interphalangeal (IP) joint, and those associated with injuries of the long fingers and palm were excluded.

The time to surgery averaged 12 to 72 hours. The patients were explained the procedure and after taking a written consent, a CFF from the ring finger dorsum was used to cover the thumb defect. The positioning of the fingers is shown in -Fig. 1.

The pedicle was divided between 2 and 3 weeks after flap transfer. One week following the division and inset of the flap, the following measurements were taken in both hands:

1. 1st web space angle in resting position, palmar abduction and in opposition to the middle finger (MF), ring finger $(\mathrm{RF})$, and little finger (LF).

2. Kapandji score

3. Total range of movement (TRM) of the long fingers (index, middle, ring and little fingers)

At 4 weeks postop, the global hand function was assessed by administering the Michigan hand outcome questionnaire (MHQ).

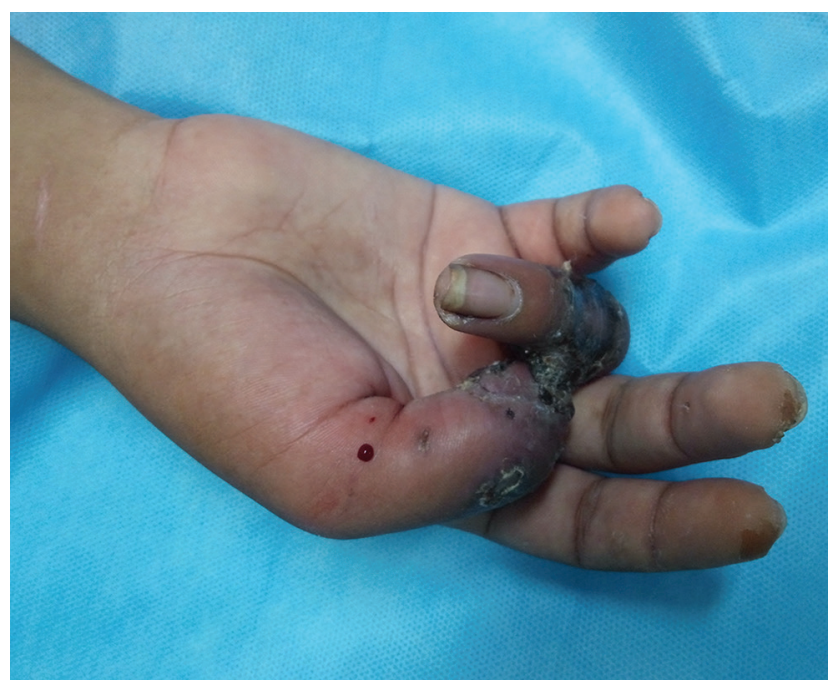

Fig. 1 Positioning of fingers.

\section{Surgical Technique}

After thorough debridement of the wound, the defect size was measured and a laterally based flap was designed by reverse planning technique on the dorsum of middle phalanx of RF. The type of CFF was chosen according to location of defect and ease of positioning. In case of dorsal defects, the flap was de-epithelialized as the reverse CFF, while for volar defects, the flap was raised without de-epithelialization. The ring finger and thumb were positioned in opposition and then the flap was inset into the defect. The secondary defect, and flap in case of reverse dermis variant were covered with a split thickness graft harvested from the same arm. ${ }^{8}$

\section{Measurements}

1. First web space angle-It is the angle subtended by the 1 st and 2nd metacarpals at the carpometacarpal joint. As there were no valid references for the standard first web space angle, 44 normal hands were measured for their first web space angles, using a goniometer, and its mean was taken as the reference value.

The angle was measured at different positions of the thumb, that is, resting position, palmar abduction, and opposition to MF, RF and LF. The mean values +/-standard deviation (SD) obtained were as follows:

- Resting-35.95 $\pm 3.12^{\circ}$

- Palmar abduction $-46.7^{\circ} \pm 3.12^{\circ}$

- Opposition to-

- MF-34.95 $\pm 4.12^{\circ}$

- $\mathrm{RF}-37.45^{\circ} \pm 4.15^{\circ}$

- LF- $42.18^{\circ} \pm 4.66^{\circ}$

In each of the cases, the web angle was measured with the flap in situ and the resting angle 1-week postdivision. For comparison, the resting angle of opposite hand was also measured.

2. Kapandji score-Utilized to assess thumb opposition, the Kapandji score ${ }^{9}$ was applied to the subjects 1 week after division of flap. The score of the affected hand was compared with the normal hand, that is, a score of 10 . The lowest score is 1 , that is, the thumb is able to touch radial side of the proximal phalanx of the IF. The highest score is 10 wherein the patient is able to touch the ulnar end of the distal palmar crease.

3. TRM of the long fingers-in both hands, especially that of the donor finger was measured using a finger goniometer. The TRM of a finger $=$ total flexion of the finger (sum of flexion at metacarpophalangeal [MCP], proximal interphalangeal [PIP] and distal interphalangeal [DIP] joints) - total extension of the finger ${ }^{10}$

4. MHQ-It is a tool used to assess patients with hand disorders through the measurement of six health domains: overall hand function, activities of daily living (ADL), pain, work performance, aesthesis, and patient satisfaction. All six domains are scored from 0 to100. Higher score indicates a better performance, except for pain where higher score indicates greater pain. The mean score for the hand 
is calculated by taking an average of scores obtained in each domain. ${ }^{11,12}$

The questionnaire was administered to the affected and the unaffected hand for each patient at the end of 4 weeks following flap division.

In case of stiffness of the affected hand, the patients were given physiotherapy and followed-up 2 weeks after the initiation of physiotherapy.

\section{Results}

A total of 10 patients were operated upon. There were nine males and one female. The mean age of the patients was 31.5 yrs. Six of the injuries were occupation-related, while four were from traffic accidents. Out of 10 cases, two defects resulted from debridement of failed thumb replants. There was an equal distribution of right and left sides. The defect size varied from 3.8 to $10 \mathrm{~cm}^{2}\left(6.71 \pm 2.32 \mathrm{~cm}^{2}\right)$. The defects were located dorsally $(n=3)$, volar $(n=3)$, and at the tip $(n=4)$. Three patients had loss of terminal phalanx, resulting in shortening of the thumb. Six patients were operated with reversed dermis CFFs, one patient with a proximally based CFF, while the rest were operated with classical CFF. The donor finger in all the patients was the RF. All patients were operated within 12 to 72 hours of injury, and the flaps were divided between the 2 nd and 3rd week after flap transfer (-Table 1).
One patient developed complete flap detachment, for which re-inset was given at 2 weeks postop and division done 3 weeks post re-inset. Three patients who developed global stiffness of operated hand were given 1 to 2 weeks of physiotherapy and measurements taken after return of flexibility.

The first web angle of the operated hand was measured with flap in situ $\left(40^{\circ} \pm 2.51^{\circ}\right)$ and was found to be slightly higher than the average resting normal first web angle $\left(35.95^{\circ} \pm 3.12^{\circ}\right)$. The first web angle measured after flap division was found to be similar to that of the unaffected side. The mean resting first-web angle of the affected hand was $36.2^{\circ} \pm 2.73^{\circ}$, and that of the unaffected hand $\left(37.3^{\circ} \pm 2.6^{\circ}\right)(p$ $=0.12$ ). The 1 st web angles of the operated hand at all positions of the thumb were similar to those of the unaffected hand.

The mean Kapandji score was 7. The lowest score was 5. This patient had P2 loss and flap detachment postflap surgery, with prolonged period of immobilization. The TRM of the IF in all the cases was maintained, except in two patients in whom there was global hand stiffness, while that of the RF was reduced with predominant reduction of movement of the DIP joint. The mean TRM of IF was $206^{\circ} \pm 5.09^{\circ}$ on the treated side and that of normal side was $204^{\circ} \pm 8.3^{\circ}(p=0.272)$; while the mean TRM of affected RF was $168.4^{\circ} \pm 17.9^{\circ}$ in comparison to the normal side $\left(205^{\circ} \pm 4.3^{\circ}\right)(p=0.000124)$ ( - Table 2$)$.

The average MHQ score for the affected hand was 64 , compared with MHQ score of the unaffected hand, that is, 72.5

Table 1 Demographic details

\begin{tabular}{|l|l|l|l|l|l|l|l|}
\hline Sr No & Age & Sex & Defect size $(\mathbf{c m})$ & Location & Bone loss & Procedure & Complications \\
\hline 1 & 38 & M & $3 \times 2.5$ & Dorsum of P2 & & Reverse dermis CFF & \\
\hline 2 & 54 & M & $2 \times 1.8$ & Dorsoradial aspect & & Reverse dermis CFF & \\
\hline 3 & 23 & M & $2.5 \times 2.8$ & Volar & + & Classical CFF & Stiffness \\
\hline 4 & 44 & M & $2.5 \times 1.5$ & Volar tip loss & + & Classical CFF & \\
\hline 5 & 17 & M & $2 \times 2.5$ & Dorsal tip loss & & Reverse dermis CFF & \\
\hline 6 & 43 & M & $3.5 \times 3$ & Volar radial aspect & & Proximally based CFF & \\
\hline 7 & 30 & F & $4 \times 2.5$ & Tip and dorsum of P1 & & Reverse dermis CFF & Stiffness \\
\hline 8 & 25 & M & $3 \times 2.5$ & Volar ulnar aspect & & Classical CFF & \\
\hline 9 & 19 & M & $2.5 \times 3.5$ & Tip of thumb & + & Reverse dermis CFF & Flap detachment \\
\hline 10 & 22 & M & $3 \times 2$ & Dorsoulnar aspect & & Reverse dermis CFF & Stiffness \\
\hline
\end{tabular}

Abbreviation: CFF, cross-finger flap.

Table 2 Total range of movement of long fingers

\begin{tabular}{|c|c|c|c|c|}
\hline \multicolumn{2}{|c|}{ Total range of movement of long fingers } & Mean & SD & $p$ value \\
\hline \multirow[t]{2}{*}{ IF } & Treated & 206.20 & 5.095 & \multirow[t]{2}{*}{0.272} \\
\hline & Normal & 204.30 & 8.301 & \\
\hline \multirow[t]{2}{*}{ MF } & Treated & 209.40 & 3.978 & \multirow[t]{2}{*}{0.200} \\
\hline & Normal & 206.50 & 6.754 & \\
\hline \multirow[t]{2}{*}{ RF } & Treated & 168.40 & 17.995 & \multirow[t]{2}{*}{$<0.0001$} \\
\hline & Normal & 205.50 & 4.301 & \\
\hline \multirow[t]{2}{*}{ LF } & Treated & 195.00 & 9.718 & \multirow[t]{2}{*}{0.015} \\
\hline & Normal & 205.10 & 4.095 & \\
\hline
\end{tabular}

Abbreviations: IF, index finger; LF, little finger; MF, middle finger; RF, ring finger; SD, standard deviation. 
(mean difference $=2.59 ; p=0.05$ ). All patients had a good score in the ADL domain (mean=80.4 \pm 10.1 ) and satisfaction domain $(81.2 \pm 5.43)$. All patients demonstrated an average score of 2 for activities involving picking up a coin, turning a key, and of 2.5 for activities like buttoning shirts. All patients were able to hold a glass as well as their normal hand (average score of 1.1), suggesting maintenance of first web space angle. The aesthesis of the donor site and flaps was acceptable to all the patients (-Table $\mathbf{3}$ ).

Here are two representative cases.

\section{Case 1}

A 54-year-old male carpenter came with avulsion injury of the left thumb during work. The defect, around $3 \times 2.5 \mathrm{~cm}$ in size, was located on the dorsum of the P2 segment of the left thumb with nail bed loss and bone exposure. There was no fracture in the thumb. A reverse dermis cross finger flap cover was given. The donor finger chosen was the RF. The CFF was divided 2 weeks later. Postoperatively, patient had a slight flexion deficit of RF but a good global hand function (-Fig. 2).

Table 3 MHQ Score evaluation

\begin{tabular}{|c|c|c|c|c|}
\hline MHQ domains & & Mean score & SD & $p$ value \\
\hline \multirow[t]{2}{*}{ Work } & Treated & 85.00 & 7.071 & \multirow[t]{2}{*}{$<0.0001$} \\
\hline & Normal & 100.00 & 0.000 & \\
\hline \multirow[t]{2}{*}{ Pain } & Treated & 9.00 & 7.379 & \multirow[t]{2}{*}{0.004} \\
\hline & Normal & 0.00 & 0.000 & \\
\hline \multirow[t]{2}{*}{ Aesthesis } & Treated & 79.60 & 23.320 & \multirow[t]{2}{*}{0.548} \\
\hline & Normal & 75.00 & 0.000 & \\
\hline \multirow[t]{2}{*}{ Satisfaction } & Treated & 81.20 & 5.432 & \multirow[t]{2}{*}{$<0.0001$} \\
\hline & Normal & 100.00 & 0.000 & \\
\hline \multirow[t]{2}{*}{ Overall ADL } & Treated & 80.400 & 10.0962 & \multirow[t]{2}{*}{0.001} \\
\hline & Normal & 90.650 & 4.9275 & \\
\hline \multirow[t]{2}{*}{ Mean MHQ score } & Treated & 64.00 & 6.650 & \multirow[t]{2}{*}{0.047} \\
\hline & Normal & 72.50 & 7.962 & \\
\hline
\end{tabular}

Abbreviations: ADL, activities of daily living; MHQ, Michigan hand outcome questionnaire; SD, standard deviation.
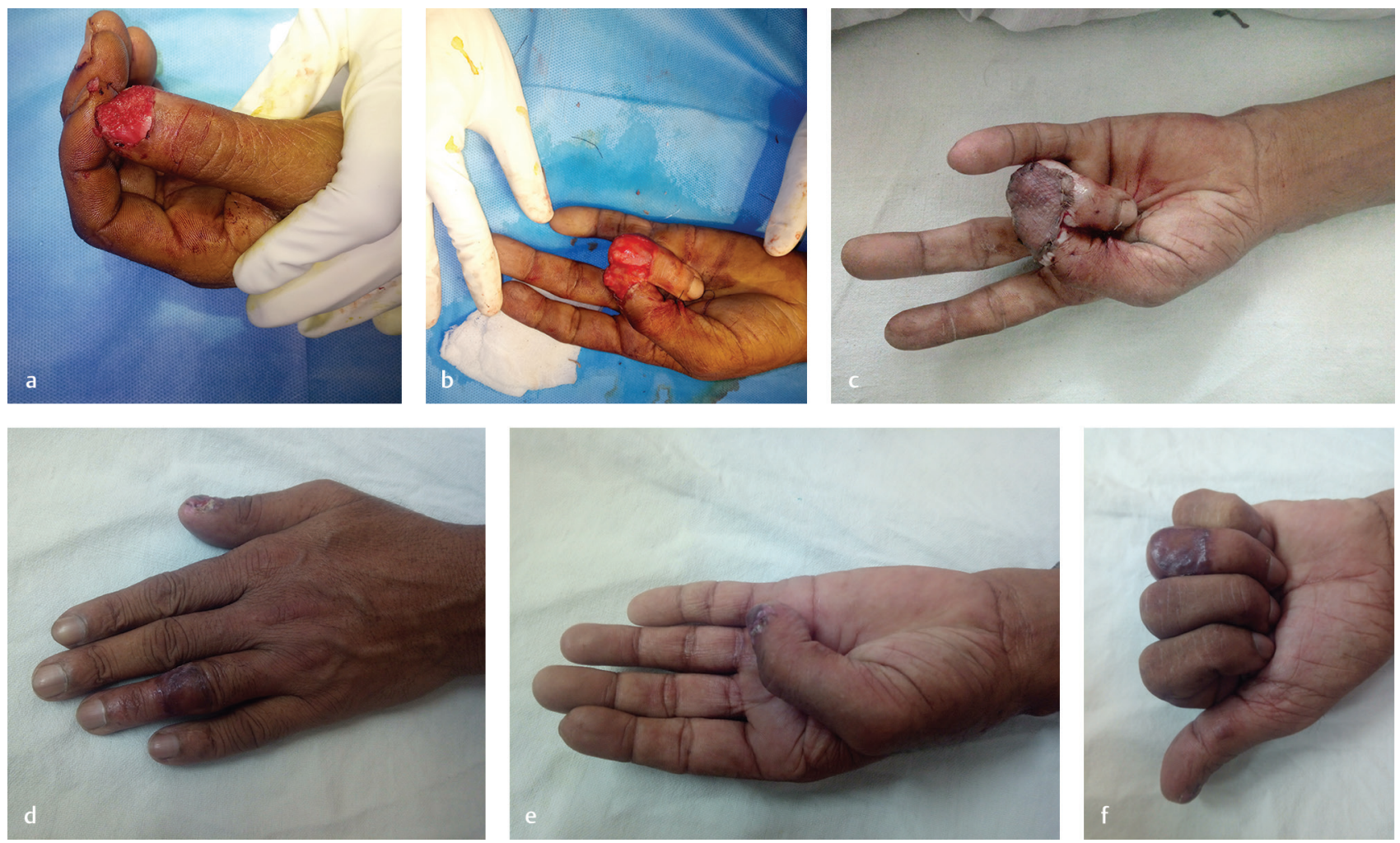

Fig. 2 Representative case-1. (a) Defect. (b) Flap inset. (c) Before division. (d) Donor site. (e) Kapandji score-9. (f) Full range of movement of donor finger. 


\section{Case 2}

A 23-year-old male clerk with near total amputation of the right thumb distal to the IP joint underwent revascularization which failed. After debridement of the thumb, there was a resultant volar defect of $2.5 \times 2.8 \mathrm{~cm}$ with bone exposure. A classical CFF cover was given from the ring finger dorsum. Secondary defect was covered with skin graft. The flap was divided 2 weeks later. Postoperatively, the patient developed global hand stiffness, which recovered with adequate physiotherapy. Patient was able to write and hold onto objects postflap division and obtained good hand function (-Fig. 3).

\section{Discussion}

The most important feature differentiating the human hand from those of the apes has been found to be opposition, allowing three types of grips-pad to side, three- jawed chuck and five-jawed cradle-chuck. The absence of this function interrupts the daily activity at a very significant level. Opposition, the hallmark of "thumbness," necessitates a painless stable skin coverage and an acceptable length to enable circumduction. ${ }^{1}$

The CFF is one versatile flap which, due to its reliability and ease of performance, has been the mainstay of management of large distal defects of the thumb. The flap had been classically used only for volar defects, but various modifications in technique, design and donor finger have made it useful for a plethora of soft tissue defects of fingers.
The CFF is predominantly based on the dorsal digital vessels. Although classically, they are raised based on the lateral border of the finger, they may be raised as proximally or distally based flaps or on a single vascular pedicle, thus allowing greater mobilization of the flap. The donor finger to the thumb is classically described as the index finger, wherein the first web space is closed to allow easy inset of the flap ${ }^{1,4}$ However, it may cause joint stiffness, first web contracture, poor local hygiene, and unsightly appearance.

Of the eight basic hand positions, all of them involves IF, and 6/8 involves MF, making them the second and third important fingers respectively after thumb. ${ }^{5}$ LF has too little area to be harvested as CFF and positioning also becomes difficult. This leaves RF as a probable choice.

Woon et al, in their study, share their experience with use of MF as donor finger for resurfacing of radial volar defects of the thumb, with no difference in the donor site morbidity as compared with those wherein IF was used as donor finger. However, they did observe that radial-based defects were better covered by flaps from long fingers other than the IF as the long finger would wrap around the injured thumb tip, thereby reducing the amount of flap tissue necessary by diminishing the redundant tissue bridge. Also, one advantage of multiple donor site possibilities is that revision surgery is easily accomplished should the original CFF fail. ${ }^{13}$ Mutaf et al, in their case series of the C- ring flap, an axial pattern CFF based either on distal or proximal digital vascular bundle, utilized the ring finger as the donor finger for thumb defects.
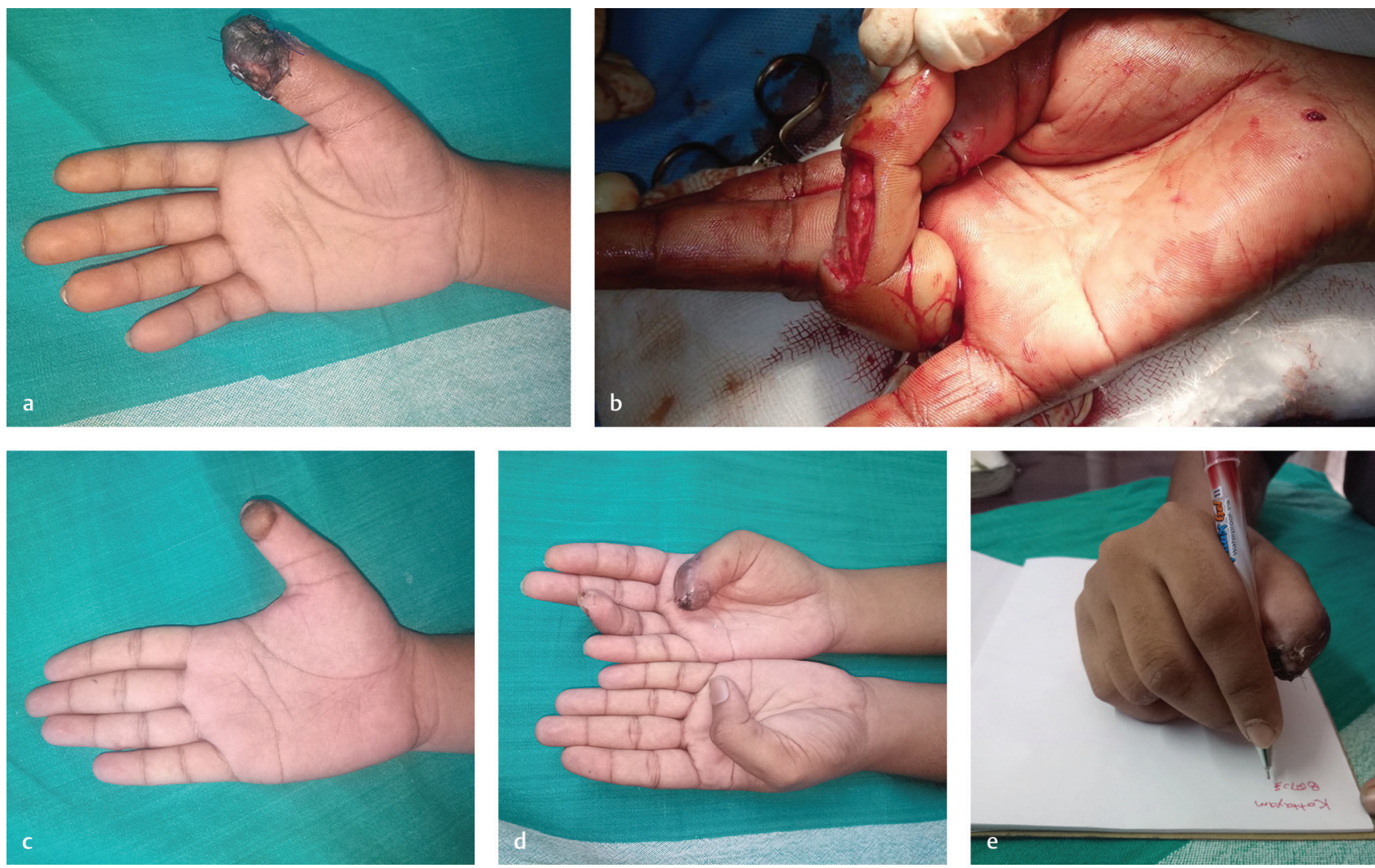

Fig. 3 Representative case-2. (a) Failed thumb tip replant. (b) CFF in situ. (c) After flap division. (d) Kapandji score-7. (e) Postop hand function. Abbreviation: CFF, cross-finger flap. 
Also, they recommended the use of the radial side of the 3rd or 4th fingers as donor fingers for thumb defects as this side of these fingers is most hidden from view. ${ }^{14}$

Koch et al demonstrated, in their study, a significant difference in the TRM between the donor finger $\left(156^{\circ}\right.$, range $70-235$ ) and control fingers ( $175^{\circ}$, range $\left.95-270\right)$. Also, a significant decrease in the maximal pinch grip strength of the donor finger to the thumb was demonstrated. ${ }^{6}$ In our study, the TRM of the donor fingers also averaged at $168.4^{\circ} \pm 17.9^{\circ}$, whereas that of the control fingers was $205.5^{\circ} \pm 4.3^{\circ}(p$ value $=0.000124)$. There was no difference in the TRM of the IF between the treated and normal hands. Since the donor morbidity was shifted to a relatively unimportant finger, global function remained less affected as has been demonstrated by the proximity of the mean MHQ scores of the affected (64 \pm $6.64)$ to that of the unaffected hand $(72.5 \pm 7.9)(p=0.05)$, and the good scores achieved, especially in domains of ADL and Work. All patients had a satisfactory overall hand function at the end of follow-up.

\section{Conclusion}

We suggest RF as an alternate donor finger for a CFF to thumb as compared with IF for the following reasons:

1. Sparing of IF and MF, which are second and third fingers in order of functionality

2. Comfortable positioning for the patient.

3. The first web angle with opposition to RF was close to the average resting angle of first web, thus preventing any first web contracture

4. Ease of maintenance of local hygiene with flap in situ.

This is a pilot study to search for alternate donor fingers other than index. This may be an inspiration for future studies with more sample size and better outcome assessment.

\section{Note}

The study has been approved by the Institutional Review Board, Government Medical College, Kottayam, Kerala. Part of this study was presented by the first author in the annual state conference of Kairali Plastic Surgeons Association (KPSACON-2019) held in February 2019.

\section{Funding}

No financial disclosure to be made by any of the authors

\section{Conflict of Interest}

None declared.

\section{References}

1 Lee KK, Thumb Reconstruction WPA In: Green's Operative Hand Surgery 6th Edition. 6th ed.;1839-1852

2 Karthikeyan GR. Versatility and modifications of CFF in hand surgery.pdf. Int J Sci Stud 2017;5(6):35-46

3 David A, Kappel JGB. The cross finger flap HC 1985 Kappel.pdf. Hand Clin 1985;1(4):677-683

4 Vedder NB, Friedrich JB. Thumb reconstruction: Nonmicrosurgical techniques. In: Peter CN (Editor in Chief), James C (Volume Editor), eds. Plastic Surgery Volume 6. 4th ed; 2018 305-306

5 Smith P, Reconstruction. In: Smith P, ed. Lister's The Hand: Diagnosis and Indications. 4th ed. Churchil Livingston; 2002 145-146

6 Koch H, Kielnhofer A, Hubmer M, Scharnagl E. Donor site morbidity in cross-finger flaps. Br J Plast Surg 2005;58(8): 1131-1135

7 Paterson P, Titley OG, Nancarrow JD. Donor finger morbidity in cross-finger flaps. Injury 2000;31(4):215-218

8 Atasoy E. The reverse cross finger flap. J Hand Surg Am 2016;41(1):122-128

9 Ghoraba SM, Mahmoud WH. Outcome of thumb reconstruction using the first dorsal metacarpal artery island flap. World J Plast Surg 2018;7(2):151-158

10 Kenney RJ, Hammert WC. Physical examination of the hand. J Hand Surg Am 2014;39(11):2324-2334, quiz 2334

11 Shauver MJ, Chung KC. The Michigan hand outcomes questionnaire (MHQ) after 15 years of field trial. Plast Reconstr Surg 2013;131(5):779e-787e

12 Chung KC, Pillsbury MS, et al. Reliability and validity testing of the Michigan Hand Outcomes Questionnaire. J Hand Surgery 1998;23A; 575-587

13 Woon CYL, Lee JYL, Teoh LC. Resurfacing hemipulp losses of the thumb: the cross finger flap revisited: indications, technical refinements, outcomes, and long-term neurosensory recovery. Ann Plast Surg 2008;61(4):385-391

14 Mutaf M, Sensöz O, Ustüner ET. A new design of the crossfinger flap: the C-ring flap. Br J Plast Surg 1993;46(2):97-104 